\title{
A THEOREM ON CONTINUOUS DECOMPOSITIONS OF THE PLANE INTO NONSEPARATING CONTINUA
}

\author{
MICHEL SMITH
}

\begin{abstract}
E. Dyer [2] proved that there is no continuous decomposition of a compact irreducible continuum into decomposable continua which is an arc with respect to its elements. The author extends Dyer's result to the plane. Consider a continuous decomposition of the plane into nonseparating compact continua. R. L. Moore [6] has shown that the decomposition space is homeomorphic to the plane. Using Moore's result it is shown that the union of the elements of each arc in the decomposition space is an irreducible continuum. It follows then, from Dyer's result, that there is no continuous decomposition of the plane into nonseparating compact decomposable continua.
\end{abstract}

In $1936 \mathrm{~J}$. H. Roberts [7] proved that there is no upper semicontinuous decomposition of the plane into arcs. In the same paper Roberts gives an example of an upper semicontinuous decomposition of the plane each element of which is an arc or an " $H$ ". In 1968 Stephen L. Jones extended Roberts' theorem to $E^{n}$ [3]. R. D. Anderson announced [1] that there exists a continuous decomposition of the plane into pseudo-arcs. In 1949 E. E. Moise [5] proved that there is no continuous decomposition of a compact irreducible continuum into arcs which is an arc with respect to its elements. E. Dyer extended this result and showed that there is no continuous decomposition of a compact irreducible continuum into decomposable continua which is an arc with respect to its elements [2].

It follows from Dyer's result and the theorem proven in this paper that there is no continuous decomposition of the plane into nonseparating compact decomposable continua.

If $G$ is a collection of point sets then $G^{*}$ means the union of the elements of $G$. If $G$ is an upper semicontinuous collection then $G^{*} / G$ means the decomposition space. The Euclidean plane is denoted by $E^{2}$.

The author wishes to thank the referee for indicating a much shorter proof of the main theorem which follows.

The following theorems are assumed; for proofs the reader should refer to [6].

THEOREM A. If $G$ is an upper semicontinuous collection of compact continua

Received by the editors April 14, 1975 and, in revised form, July 21, 1975.

AMS (MOS) subject classifications (1970). Primary 54B15.

Key words and phrases. Continuous decomposition, atomic decomposition, decomposable continuum, $E^{2}$. 
which fills up $E^{2}$ and no one of them separates $E^{2}$, then the decomposition space $G^{*} / G$ is homeomorphic to $E^{2}$.

THEOREM B (JANISZEWSKI). If $H$ and $K$ are two continua (closed connected sets) and $\beta$, the boundary of $H \cap K$, is compact and $H \cap K$ is not connected, then $H \cup K$ separates $E^{2}$.

THEOREM. Suppose that $G$ is a continuous collection of compact continua which do not separate the plane and $G^{*}=E^{2}$. Then if $L$ is an arc in $G^{*} / G, L^{*}$ is an irreducible continuum in $E^{2}$.

Proof. The decomposition space $G^{*} / G$ is homeomorphic to $E^{2}$ (Theorem A). Let $L$ be an arc in $G^{*} / G$ with endpoints $g_{A}$ and $g_{B}$ and let $C$ be a simple closed curve in $G^{*} / G$ which contains $L$. Then $G^{*} / G-L$ is the sum of two mutually exclusive connected domains $D_{1}$ and $D_{2}$.

Suppose $A$ and $B$ are points of $g_{A}$ and $g_{B}$ respectively, and $V$ is a proper subcontinuum of $L^{*}$ containing $A$ and $B$. Since $\left(L-\left\{g_{A}, g_{B}\right\}\right)^{*}=L^{*}$, there is a point $x$ of $L^{*}-V$ which does not belong to $g_{A}$ or $g_{B}$.

Now each point of $C^{*}$ is a limit point of $D_{1}^{*}$ and of $D_{2}^{*}$. But $\overline{C-L^{*}}$ and $V$ are two continua whose common part is not connected and so by Theorem B, $V \cup \overline{C-L^{*}}$ separates two points, $p$ and $q$, of $E^{2}$. But $D_{1}^{*} \cup\{x\} \cup D_{2}^{*}$ is a connected set having $p$ and $q$ as limit points. Thus $D_{1}^{*} \cup\{x\} \cup D_{2}^{*} \cup\{p\}$ $\cup\{q\}$ is a connected subset of $E^{2}-\left(V \cup \overline{C-L^{*}}\right)$ containing both $p$ and $q$. This is a contradiction so the theorem is true.

Suppose that $G$ is an upper semicontinuous collection. William S. Mahavier [4] defines the following

Property A. If $g \in G$, each point of $g$ is a limit point of the union of the members of each component of $G-g$.

Corollary. Suppose that $G$ is an upper semicontinuous collection of compact plane continua no one of which separates the plane, $G^{*}=E^{2}$ and if $L$ is an arc in $G^{*} / G$ then $L$ has Property A. Then some element of each arc in $G^{*} / G$ is indecomposable.

Proof. The proof follows from the argument above and Theorem 4 of [4].

\section{REFERENCES}

1. R. D. Anderson, Open mappings of compact continua, Proc. Nat. Acad. Sci. U.S.A. 42 (1956), 347-349. MR 17, 1230.

2. E. Dyer, Irreducibility of the sum of the elements of a continuous collection of continua, Duke Math. J. 20 (1953), 589-592. MR 15, 335.

3. Stephen L. Jones, The impossibility of filling $E^{n}$ with arcs, Bull. Amer. Math. Soc. 74 (1968), 155-159. MR 36 \#5917.

4. W. S. Mahavier, Upper semi-continuous decompositions of irreducible continua, Fund. Math. 60 (1967), 53-57. MR 34 \#388.

5. E. E. Moise, $A$ theorem on monotone interior transformations, Bull. Amer. Math. Soc. 55 (1949), 810-811. MR 11, 121.

6. R. L. Moore, Foundations of point set theory, rev. ed., Amer. Math. Soc. Colloq. Publ., vol. 13, Amer. Math. Soc., Providence, R.I., 1962. MR 27 \# 709.

7. J. H. Roberts, There does not exist an upper semi-continuous decomposition of $E^{2}$ into arcs, Duke Math. J. 2 (1936), 10-19. 